\section{Relative Truth Degree of Logic Formulas and Graded Fuzzy Logic}

\author{
$\mathrm{Li} \mathrm{Fu}$ \\ Department of Mathematics, Qinghai Nationality College, Xining 810007, China \\ Tel: 86-971-829-2658Ｅ-mail: f10971@163.com
}

The research is financed by The Education department chunhui program (Z2006-1-81001)

\begin{abstract}
The concept of logic proposition induced functions is proposed in the present paper, then the concept of relative truth degree of propositions with respect to a logic theory $\Gamma$ is introduced by means of infinite product of evenly distributed probability spaces and integrated semantics respectively w.r.t. discrete and continuous situations, and a graded approximate reasoning theory is established. Next, theory of consistency degrees of finite logic theories is also proposed. Finally, the simple application of graded fuzzy logic in fuzzy inference is given by examples.
\end{abstract}

Keywords: Logic proposition induced functions, Truth degree of propositions, Approximate reasoning, Consistency degree of finite logic theories

\section{Preliminaries}

Let $S=\left\{p_{1}, p_{2}, \cdots\right\}$ be a countable set, and $\overline{0}$ be a special element not contained in $S$, and $F(S)$ be the free algebra of type $(\&, \vee, \wedge, \rightarrow)$ generated by $S \cup\{\overline{0}\}$, where $\&, \vee, \wedge, \rightarrow$ are binary operations respectively, i.e.,

(i) $S \subset F(S), \overline{0} \in F(S)$

(ii) If $A, B \in F(S)$, then $A \& B, A \vee B, A \wedge B$, and $A \rightarrow B \in F(S)$

(iii) Elements of $F(S)$ can be obtained by (i)and (ii) in a finite step of calculus.

Members of $S$ are called atomic propositions or atomic formulas (briefly, atoms), that of $F(S)$ are propositions or formulas or wffs. $\overline{0}$ is called contradiction. Moreover, in what follows $\neg A=A \rightarrow \overline{0}$. For classical two valued propositional logic system $L$, the following are axiom schemes.

(L1) $A \rightarrow(B \rightarrow A)$

(L2) $(A \rightarrow(B \rightarrow C)) \rightarrow((A \rightarrow B) \rightarrow(A \rightarrow C))$

(L3) $(\neg A \rightarrow \neg B) \rightarrow(B \rightarrow A)$

The deduction rule is modus ponens (MP), i.e., from $A \rightarrow B$ and $A, B$ follows. The following theorem holds in $L$ (Ref A.G. Hamilton.( 1979)):

Theorem 1 In classical propositional logic system $L$, suppose that $\Gamma \subset F(S), A, B \in F(S)$, and $\Gamma \bigcup\{A\} \vdash B$, then $\Gamma \mid-A \rightarrow B$, and vice versa. In $L$, we have $A \vee B=\neg A \rightarrow B, A \& B=A \wedge B$

$=\neg(\neg A \vee \neg B)$.

In the fuzzy prepositional logic system $L^{*}$ the following are axiom schemes

(L*1) $\quad A \rightarrow(B \rightarrow A \wedge B)$

$\left(L^{*} 2\right) \quad(\neg A \rightarrow \neg B) \rightarrow(B \rightarrow A)$

(L'3) $\quad(A \rightarrow(B \rightarrow C)) \rightarrow(B \rightarrow(A \rightarrow C))$

$\left(L^{*} 4\right) \quad(B \rightarrow C) \rightarrow((A \rightarrow B) \rightarrow(A \rightarrow C))$

(L) $\quad A \rightarrow \neg \neg A$ 
(L) $\quad A \rightarrow A \vee B$

$\left(L^{*} 7\right) \quad A \vee B \rightarrow B \vee A$

$\left(L^{*} 8\right) \quad(A \rightarrow C) \wedge(B \rightarrow C) \rightarrow(A \vee B \rightarrow C)$

$\left(L^{*} 9\right) \quad(A \wedge B \rightarrow C) \rightarrow(A \rightarrow C) \vee(B \rightarrow C)$

$\left(L^{*} 10\right) \quad(A \rightarrow B) \vee((A \rightarrow B) \rightarrow \neg A \vee B)$

where $A \vee B=\neg\left((((A \rightarrow(A \rightarrow B)) \rightarrow A) \rightarrow \neg((A \rightarrow B) \rightarrow B)), A \wedge B=\neg(\neg A \vee \neg B)\right.$. The deduction rule in $L^{*}$ is also MP, and we have the following

Theorem 2 Suppose that $\Gamma \subset F(S), A, B \in F(S), \quad \Gamma \cup\{A\} \vdash B$, then $\Gamma \vdash A^{2} \rightarrow B$, and vice versa, where $A^{2}=A \& A=\neg(A \rightarrow \neg A)$.

In $\quad L^{*} \quad$ we $\quad A \wedge B=\neg(\neg A \vee \neg B)$

$A \& B=\neg(A \rightarrow \neg B), \quad$ and $\quad A \vee B=\neg((((A \rightarrow(A \rightarrow B)) \rightarrow B) \rightarrow B) \rightarrow \neg((A \rightarrow B) \rightarrow B)) \quad$ (see $\quad$ Ref G.J.Wang( 2003),S.M.Wang, etc(2003)).

\section{Truth degrees of propositions in $L$ and $L^{*}$}

Let $A=A\left(p_{1}, \cdots, p_{n}\right)$ be a formula in $F(S)$, then $A$ induced a function $\bar{A}\left(x_{1}, \cdots, x_{n}\right): V^{n} \rightarrow V$, where $V=\{0,1\}$ or $V=[0,1]^{n}$, as follows: the value of $\bar{A}\left(x_{1}, \cdots, x_{n}\right)$ can calculated in $V$ by connecting $x_{1}, \cdots, x_{n}$ with the operators

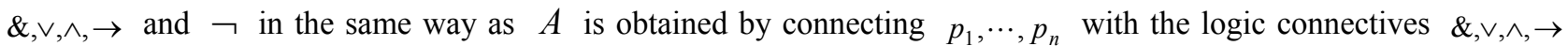
and $\neg$ respectively. For example, if $A=\left(p_{1} \rightarrow p_{2}\right) \rightarrow \neg p_{3} \vee p_{4}$, then $\bar{A}\left(x_{1}, x_{2}, x_{3}, x_{4}\right)=\left(x_{1} \rightarrow x_{2}\right) \rightarrow\left(1-x_{3}\right) \vee x_{4}$. In case $\quad v=\{0,1\}, \bar{A}(0,0,1,0)=\quad(0 \rightarrow 0) \rightarrow(1-1) \vee 0=0 \rightarrow 0=1, \quad$ and $\quad$ in $\quad$ case $\quad V=[0,1]$, $\bar{A}(0.8,0.3,0.9,0.2)=(0.8 \rightarrow 0.3) \rightarrow(1-0.9) \vee 0.2 \quad=0.3 \rightarrow 0.2=0.7, \quad$ where $\rightarrow \quad$ is the $R_{0}$-implication operator, $R_{0}=a \rightarrow b=\left\{\begin{array}{l}1, a \geq b \\ (1-a) \vee b, a>b\end{array}\right.$, ( see Ref G.J.Wang. $\left.(2003,1998)\right)$.

In the following $\bar{A}\left(x_{1}, x_{2}, \ldots x_{n}\right): V^{n} \rightarrow V$ will be called the proposition $A$ induced function.

Since $F(S)$ is the free algebra generated by $S \cup\{\overline{0}\}$ and for every valuation $v: F(S) \rightarrow V, v(\overline{0})=0$ holds, hence $v$ is determined by $v \mid S$, i.e., $v$ is determined by $\left(v\left(p_{1}\right), v\left(p_{2}\right), \cdots\right) \in V^{\infty}$. Let $\mu$ be the infinite product of evenly distributed probability measure on $V=\{0,1\}$, and $\Omega$ be the set consisting of all valuations of $F(S)$, then there is a one-one correspondence between $\Omega$ and $\{0,1\}^{\infty}$. In fact, assume that $v\left(p_{k}\right)=v_{k}(k=1,2, \ldots)$, then $\vec{v}=\left(v_{1}, v_{2}, \ldots\right) \in[0,1]^{\infty}$.

Conversely, assume that $\vec{v}=\left(v_{1}, v_{2}, \ldots\right) \in[0,1]^{\infty}$, then there exists an unique $v \in \Omega$, such that $v\left(p_{k}\right)=v_{k},(k=1,2, \ldots)$, hence $\varphi: \Omega \rightarrow\{0,1\}^{\infty}$ is a bijection, where $\varphi(v)=\vec{v}$.

Let $\varphi: \Omega \rightarrow\{0,1\}^{\infty}$ be the bijection, then $\forall A \in F(S)$, define $[A]$ and $\tau(A)$ as follows respectively: $[A]=\{\vec{v} \in X \mid v \in \Omega, v(A)=1\}, \tau(A)=\mu([A]), \tau(A)$ is called the truth degree of $A$. It is clear that $0 \leq \tau(A) \leq 1$

In case $V=[0,1], \forall A \in F(S), \operatorname{call}_{\tau}(A)=\int_{\Delta} \bar{A}\left(x_{1}, \cdots, x_{n}\right) d w=\int_{0}^{1} \cdots \int_{0}^{1} \bar{A}\left(x_{1}, \cdots, x_{n}\right) d x_{1} \cdots d x_{n}$

integrated truth degree of $A$, where $\Delta=[0,1]^{n}$ and $d w=d x_{1} \cdots d x_{n}$ whenever $A=A\left(p_{1}, \cdots, p_{n}\right)$. Specifically, we can define integrated truth degree of $A$ with respect to diverse implication operators. In reference P.H'ajek(1998), you can the following definition:

Definition 1 Suppose that $A\left(p_{1}, \cdots, p_{n}\right)$ is a formula in $L^{*}$,define

$\tau_{R}(A)=\int_{0}^{1} \cdots \int_{0}^{1} \bar{A}\left(x_{1}, \cdots, x_{n}\right) d x_{1} \cdots d x_{n}$, then $\tau_{R}(A)$ is called integrated truth degree of $\mathbf{A}$ with respect to implication operator R.

Example $1 \tau(p)=\int_{0}^{1} x d x=\frac{1}{2}$

$\tau(p \vee q)=\int_{0}^{1} \int_{0}^{1}(x \vee y) d x d y=\int_{0}^{1} \int_{y}^{1} x d x d y+\int_{0}^{1} \int_{x}^{1} y d x d y=\frac{1}{3}+\frac{1}{3}=\frac{2}{3}$ 


$$
\begin{aligned}
& \tau(p \wedge q)=\int_{0}^{1} \int_{0}^{1}(x \wedge y) d x d y=\int_{0}^{1} \int_{y}^{1} y d x d y+\int_{0}^{1} \int_{x}^{1} x d x d y=\frac{1}{3} \\
& \tau(p \wedge \neg p)=\int_{0}^{1}\left(x \wedge(1-x) d x=\int_{0}^{\frac{1}{2}}(1-x) d x+\int_{\frac{1}{2}}^{1} x d x=\frac{3}{4}\right. \\
& \tau\left(p_{1} \wedge \cdots \wedge p_{n}\right)=\int_{0}^{1} \int_{x_{1}}^{1} x_{1} d x d y+\cdots+\int_{0}^{1} \int_{x_{n}}^{1} x_{n} d x d y=\frac{1}{n+1}, \tau\left(p_{1} \vee \cdots \vee p_{n}\right)=\frac{n}{n+1} \\
& \tau(p \rightarrow p)=\int_{0}^{1} \int_{y}^{1}(1-x+y) d x d y+\int_{0}^{1} \int_{x}^{1} d x d y=\frac{1}{3}+\frac{1}{2}=\frac{5}{6}, \text { where } \rightarrow \text { is Lukasiewicz }
\end{aligned}
$$

implication operator $R_{L u}(a, b)=(1-a+b) \wedge 1, a, b \in[0,1]$.

Example 2 Suppose that $A=p_{1} \rightarrow\left(\neg p_{2} \rightarrow \overline{0}\right)$, let $\rightarrow$ be $R_{0}$ implication operator, then $\tau(A)=\int_{[0,1]^{2}}\left(x_{1} \rightarrow x_{2}\right) d x_{1} d x_{2}=\int_{[0,1]^{2}}(x \rightarrow y) d x d y$. Divide $[0,1]^{2}$ into 3 parts as follows

$\Delta_{1}=\left\{\left|(x, y) \in[0,1]^{2}\right| x \leq y\right\}, \Delta_{2}=\left\{\left|(x, y) \in[0,1]^{2}\right| y<x, 1-y \leq x\right\}$

$$
\Delta_{3}=\left\{\left|(x, y) \in[0,1]^{2}\right| y<x<1-y\right\}
$$

Then $[0,1]^{2}=\Delta_{1} \cup \Delta_{2} \cup \Delta_{3}$, and $\Delta_{1}, \Delta_{2}, \Delta_{3}$ are

disjoint

from

other, $\tau(A)=\int_{\Delta_{1}} 1 d x d y+\int_{\Delta_{2}}((1-x) \vee y) d x d y+\int_{\Delta_{3}}((1-x) \vee y) d x d y=\frac{1}{2}+\int_{\Delta_{2}} y d x d y+\int_{\Delta_{3}}(1-x) d x d y=\frac{1}{2}+\frac{1}{8}+\frac{1}{8}=\frac{3}{4}$.

each

\section{Graded approximate reasoning theory}

Suppose that $V=\{0,1\}, \Gamma$ is a finite theory in $L$, i.e., $\Gamma$ is a finite subset of $F(S)$, say, $\Gamma=\left\{A_{1}, \cdots, A_{n}\right\}$, and $B \in F(S)$.

Definition 2 Define $\tau(\Gamma \vdash B)=\tau\left(A_{1} \rightarrow\left(A_{2} \rightarrow \cdots\left(A_{n} \rightarrow B\right) \cdots\right)\right)$, call $\tau(\Gamma \vdash B)$ truth degree of $B$ relative to $\Gamma$ of the fact that $\Gamma$ implies $B$ in $L$, simply, the truth degree $\Gamma \vdash B$.

Theorem $3 \Gamma \vdash B$ holds if and only if $\tau(\Gamma \vdash B)=1$.

Proof Assume that $\Gamma \vdash B$ holds, then it follows from the deduction theorem of $L$ that $\tau\left(A_{1} \rightarrow\left(A_{2} \rightarrow \cdots\left(A_{n} \rightarrow B\right) \cdots\right)\right)$ is a theorem, hence it follows from Definition 1 that $\tau(\Gamma \vdash B)=1$. Conversely, assume that $\tau(\Gamma \vdash B)=1$, we can prove $\Gamma \vdash B$ similarly.

Theorem 3 told us from $\Gamma$ can imply $B$, then $\Gamma$ implies $B$ is a tautology, i.e., its truth degree equals 1 in $L$.

Example 3 If $\Gamma=\{p \rightarrow q, \neg q \rightarrow r\}$, and $B=\neg p$, then $\tau(\Gamma \mid-B)=(\tau(p \rightarrow q) \rightarrow$

$((\neg q \rightarrow r) \rightarrow \neg p))=\frac{3}{4}$, in fact, there are 8 possible $(p, q, r)$ in $\{0,1\}^{3}$ and only 2 of them, i.e., $(1,1,0)$ and $(1,1,1)$ imply that $(p \rightarrow q) \rightarrow((\neg p \rightarrow r) \rightarrow \neg p)$ equals 0 .

Suppose that $V=[0,1]$ and $\Gamma=\left\{A_{1}, \cdots, A_{n}\right\}$, and $B$ are finite theory and a formula in $L^{*}$ respectively, and $L^{*}$ is complete ( Ref D.W.Pei,etc(2002))

Definition 3 Define $\tau(\Gamma \vdash B)=\tau\left(A_{1}^{2} \rightarrow\left(A_{2}^{2} \rightarrow \cdots\left(A_{n}^{2} \rightarrow B\right) \cdots\right)\right)$, and call $\tau(\Gamma \vdash B)$ truth degree of the fact that $\Gamma$ implies $B$ in $L^{*}$.

It is similar with theorem 3 , for integrated truth degrees of formulas, we have:

Theorem 4 If $\Gamma \vdash B$ holds, then $\tau(\Gamma \vdash B)=1$. Conversely, if $\tau(\Gamma \vdash B)=1$, then $E=A_{1}^{2} \rightarrow\left(A_{2}^{2} \rightarrow \cdots\left(A_{n}^{2} \rightarrow B\right) \cdots\right)$ is an almost tautology, i.e., the induced function $\bar{E}$ of $E$ equals 1 almost every where in $[0,1]^{m}$, assume $E$ contains $m$ atomic propositions.

Proof Assume that $\Gamma \vdash B$ holds, then it follows from Theorem 2 that

$E=A_{1}^{2} \rightarrow\left(A_{2}^{2} \rightarrow \cdots\left(A_{n}^{2} \rightarrow B\right) \cdots\right)$ is a theorem of $L^{*}$, hence it follows from Definition 2 that $\tau(\Gamma \vdash B)=1$. Conversely, assume that $\tau(\Gamma \vdash B)=1$, then it follows from the definition of integrated truth degree of $E$ that the integral of $\bar{E}$ equals 1 , hence the function $\bar{E}$ equals 1 almost everywhere in $[0,1]^{m}$.

Theorem 4 means the theory of truth degrees of propositions is uniform with deduction theorem of finite theories in $L^{*}$. 
This fact is significant, it can be used to discuss deduction theorem by truth theory, and to propose a theory of consistency degrees of finite theories in $L^{*}$ in section 5 , except that the concept of truth degrees of formulas in two valued logic has to be substituted by the concept of integrated truth degrees of formulas.

Example 4 If $\Gamma=\{p\}, B=\neg q$, then it follows from the basic properties of $R_{0}$-implication operator that, $\tau(\Gamma \mid-B)=\int_{0}^{1} \int_{0}^{1}(x \otimes x \rightarrow(1-y)) d x d y=\frac{19}{24}$, where $\otimes:[0,1]^{2} \rightarrow[0,1]$ is a $t$-norm such that $(\otimes, \rightarrow)$ is a residuated pair,(see Ref G.J.Wang,etc(2003)), that is, $a \otimes b=\left\{\begin{array}{l}a \wedge b, a+b>1 \\ 0, a+b \leq 1\end{array}\right.$, and hence $a \otimes a=\left\{\begin{array}{l}a, a>\frac{1}{2} \text {. } \\ 0, a \leq \frac{1}{2}\end{array}\right.$

In [9] a pseudo-metric was defined on $\mathrm{F}(\mathrm{S})$ in $L$ as follows:

$\rho(A, B)=\int_{0}^{1} \cdots \int_{0}^{1}\left|\bar{A}\left(x_{1}, \cdots, x_{n}\right)-\bar{B}\left(x_{1}, \cdots, x_{n}\right)\right| d x_{1} \cdots d x_{n}$, where $x_{1}, \cdots, x_{n}$ are the atoms simultaneously appeared in $A$ and $B$, and $\rho(A, B)$ even $A$ and $B$ can contain different atoms, can use extensions $A^{*}$ and $B^{*}$ instead of $A$ and $B$ correspondingly, such that $A^{*}$ and $B^{*}$ contain one and the same group of atoms. e.g., if $A=p_{1}, B=p_{2}$ then let

$A^{*}=\left(p_{1} \vee p_{2} \rightarrow p_{1} \vee p_{2}\right) \rightarrow p_{1}, B^{*}=\left(p_{1} \vee p_{2} \rightarrow p_{1} \vee p_{2}\right) \rightarrow p_{2}$, we see that $A^{*} \square A, B^{*} \square B$.

Definition 5 Let $A, B \in F(S), \xi_{R}(A, B)=\int_{\Delta} R(\bar{A}, \bar{B}) \wedge R(\bar{B}, \bar{A}) d w$ is called $R$ - integral similar degree between $A$ and $B$, where $d w=d x_{1} \cdots d x_{n}$ and $\Delta=[0,1]^{n}, R$ is implication operator. If $\xi_{R}(A, B)=1$, then $A$ and $B$ is $R$-integral similar. Such, we have $\rho(A, B)=1-\int_{\Delta}(\bar{A} \rightarrow \bar{B}) \wedge(\bar{B} \rightarrow \bar{A}) d w=1-\xi_{R}(A, B)$. Furthermore, by the concept of truth degrees of formulae of $F(S)$, in $L^{*}$, a pseudo-metric can be defined as follows: $\rho(A, B)=1-\tau(A \rightarrow B) \wedge \tau(B \rightarrow A), A, B \in F(S)$.

\section{Consistency degrees of finite theories}

It is well known that a theory $\Gamma$ (i.e., a set of wffs) is consistent if the contradiction $\overline{0}$ is not a conclusion of $\Gamma$, i.e., $\Gamma \vdash \overline{0}$ does not hold, otherwise $\Gamma$ is inconsistent. Hence we see that

if $\Gamma \vdash \overline{0}$ is (fully) true, then $\Gamma$ is (fully) inconsistent.

Suppose that we can in certain way measure the truth degree, say, $\alpha$, of " $\Gamma \vdash \overline{0}$ ", then it is natural to call $\alpha$ the inconsistency degree of $\Gamma$, and the consistency degree of $\Gamma$ can then be defined to be $1-\alpha$. In this sub-section we propose the concept of consistency degrees of finite theories in accordance with this idea.

Definition 6 Suppose that $\Gamma=\left\{A_{1}, \cdots, A_{n}\right\}$ is a finite theory in $L$, let $\Gamma \vdash \overline{0}=A_{1} \rightarrow\left(A_{2} \rightarrow\left(\cdots\left(A_{n} \rightarrow \overline{0}\right) \cdots\right)\right)$, and define $\xi(\Gamma)=1-\tau(\Gamma \rightarrow \overline{0})=1-\tau\left(A_{1} \rightarrow\left(A_{2} \rightarrow\left(\cdots\left(A_{n} \rightarrow \overline{0}\right) \cdots\right)\right)\right)$,

then $\xi(\Gamma)$ is called the consistency degree of $\Gamma$.

Example 5 (i) Suppose that $p$ is an atomic formula, then $\xi(p)=1-\tau(p \rightarrow \overline{0})=1-\tau(\neg p)=1-\frac{1}{2}=\frac{1}{2}, \xi(\neg p)=1-\tau(\neg p \rightarrow \overline{0})=1-\tau(p)=1-\frac{1}{2}=\frac{1}{2}$,

(ii) Since $\tau((p \rightarrow q) \rightarrow q)=\frac{3}{4}, \tau((p \rightarrow q) \rightarrow(q \rightarrow \overline{0}))=\frac{1}{2}$, hence it follows that

$\xi(\{p \rightarrow q, q \rightarrow \overline{0}\})=1-\tau((p \rightarrow q) \rightarrow q)=\frac{1}{4}, \quad$ and $\quad \xi(\{p \rightarrow q, q\})=1-\tau((p \rightarrow q) \rightarrow(q \rightarrow \overline{0}))=\frac{1}{2} \quad$. The consistency degree of $\Gamma=\{p, q\}$ in $L$ is

$\xi(\Gamma)=1-\tau(\Gamma \rightarrow \overline{0})=1-\tau(p \rightarrow(q \rightarrow \overline{0}))=\int_{0}^{1} \int_{0}^{1}(x \rightarrow(y \rightarrow 0)) d x d y=1-\frac{3}{4}=\frac{1}{4}$, where $\rightarrow$ is

$R_{0}$ implication.

Based on theorem 2, we can propose a theory of consistency degrees of finite theories in $L^{*}$ by truth degrees of formulas.

Definition 7 Suppose that $\Gamma=\left\{A_{1}, \cdots, A_{n}\right\}$ be a finite theory in $L^{*}$, let $\left.\Gamma \vdash \overline{0}=A_{1}^{2} \rightarrow\left(A_{2}^{2} \rightarrow \cdots\left(A_{n}^{2} \rightarrow \overline{0}\right) \cdots\right)\right)$, and define 
$\xi(\Gamma)=1-\tau(\Gamma \vdash \overline{0})=1-\tau\left(A_{1}^{2} \rightarrow\left(A_{2}^{2} \rightarrow \cdots\left(A_{n}^{2} \rightarrow \overline{0}\right) \cdots\right)\right)$, then $\xi(\Gamma)$ is called the consistency degree of $\Gamma$.

Example 6 The consistency degree of $\Gamma=\{p, q\}$ in $L^{*}$ is $1-\tau\left(p^{2}-\left(q^{2} \rightarrow \overline{0}\right)\right)$, Since $\tau\left(p^{2} \rightarrow\left(q^{2} \rightarrow \overline{0}\right)\right)=\int_{0}^{1} \int_{0}^{1}\left(x^{2} \rightarrow\left(y^{2} \rightarrow 0\right) d x d y=\frac{3}{4}\right.$, hence $\xi(\Gamma)=\frac{1}{4}$.

\section{The simple application of graded fuzzy logic}

We can use the ideas of graded fuzzy logic to discuss the simple application of the triple $I$ method in graded fuzzy logic. Suppose that $A, B, A^{*}$ are different propositions of $F(S)$, the following deduction rule is called generalized modus ponens (briefly, GMP),(Ref P.H'ajek(1998)):

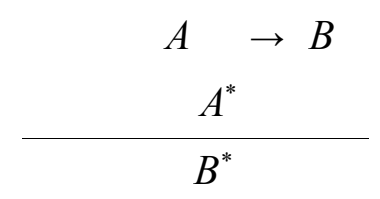

Zadeh first investigated GMP-like problem where $A, A^{*}$ and $B, B^{*}$ were supposed to be fuzzy subsets $A(x), A^{*}(x)$ and $B(y), B^{*}(y)$ on universes $X$ and $Y$ respectively, and the Compositional Rule of Inference (briefly, CRI) was proposed and the conclusion $B^{*}$ can be calculated by the following formula(Ref G.J.Wang. $(2003,2005))$

$B^{*}(y)=\sup \left\{A^{*}(x) \wedge R_{Z}(A(x), B(y)) \mid x \in X\right\}, y \in Y$

where $R_{Z}$ is Zadeh's implication operator such that

$R_{Z}(a, b)=(1-a) \vee(a \wedge b), a, b \in[0,1]$

Professor Wang proposed Triple $I$ method and it's generated form $\alpha$-triple $I$ method to improve the CRI method, where conclusion $B^{*}$ is the smallest fuzzy subset of $Y$ satisfying the condition that

$(A(x) \rightarrow B(y)) \rightarrow\left(A^{*}(x) \rightarrow B^{*}(y)\right)=1, x \in X, y \in Y$

where $\rightarrow$ is any implication operator having an adjoint $t$-norm (i.e., $a \otimes b \leq c$ if and only if

$a \leq b \rightarrow c)$, and $B^{*}$ can then be computed by the formula :

$B^{*}(y)=\sup \left\{A^{*}(x) \otimes(A(x) \rightarrow B(y)) \mid x \in X\right\}, y \in Y$

and for considering $\alpha$ restriction

$(A(x) \rightarrow B(y)) \rightarrow\left(A^{*}(x) \rightarrow B^{*}(y)\right) \geq \alpha, x \in X, y \in Y$

then $B^{*}$ can then be computed by the formula

$B^{*}(y)=\sup \left\{A^{*}(x) \otimes(A(x) \rightarrow B(y)) \mid x \in X\right\} \wedge \alpha, y \in Y$

(4) and (6) also mean the truth of formula $(A(x) \rightarrow B(y)) \rightarrow\left(A^{*}(x) \rightarrow B^{*}(y)\right)$ equals 1 and over than $\alpha$, (5)and (7) are $R_{0}$-triple $I$ and $R_{0}-\alpha$-triple $I$ algorithm respectively.

Example 5 Let $X=Y=[0,1], A, A^{*} \in F(X) B, B^{*} \in F(Y), A(x)=\frac{x+1}{4}, B(y)=1-y$,

$A^{*}(x)=1-x, \alpha=\frac{5}{9}$, let $\rightarrow$ be $R_{0}$ implication, using Eq.(5), compute $B^{*}(y)=\frac{3}{4}$, and using Eq.(7), compute $B^{*}(y)=\left\{\begin{array}{l}\frac{5}{9}, y \leq \frac{3}{4} \\ \frac{3}{4}, y>\frac{3}{4}\end{array}\right.$.

Conversely, for it's dual forms FMT, that is

$A \rightarrow B$
$B^{*} \rightarrow$

$A^{*}$ can then be computed by the formula 


$$
A^{*}(y)=\inf \left\{B^{*}(x) \vee(A(x) \rightarrow B(y)) \mid x \in X\right\}, y \in Y,
$$

and for considering $\alpha$ restriction, then $A^{*}$ can then be computed by the formula

$A^{*}(y)=\inf \left\{B^{*}(x) \vee(A(x) \rightarrow B(y)) \mid x \in X\right\} \vee \alpha^{\prime}, y \in Y$,

$A \rightarrow B$ (9) and (10) are $R_{0}$-triple $I$ and $R_{0}-\alpha$-triple $I$ MT algorithm respectively.

Example 6 Let $X=Y=[0,1], A, A^{*} \in F(X) B, B^{*} \in F(Y), A(x)=\frac{x+1}{4}, B(y)=1-y$

$B^{*}(y)=\left\{\begin{array}{l}1, y \leq \frac{3}{4} \\ \frac{3}{4}, y>\frac{3}{4}\end{array}, \alpha=\frac{5}{9}\right.$, let $\rightarrow$ be $R_{0}$ implication, using Eq.(9), compute $A^{*}(x)=\inf \varnothing=1$, and using Eq.(10), compute $A^{*}(x)=\inf \varnothing \vee \alpha^{\prime}=1 \vee \frac{4}{9}=1$.

Remark From example 5, we can see if, $A(x)=\frac{x+1}{4}, B(y)=1-y$ and $A^{*}(x)=1-x$, the minimum $B^{*}(y)$ is given by example 5 such that Eq.(5) holds, moreover, form example 6, if $A, B, B^{*}$ do not vary, let $A^{*}=1$, then by Eq.(5), $B^{*}$ is same as before. It means by Eq.(5),correspondence $\left(A, B, A^{*}\right) \rightarrow B^{*}$ is more to one.

\section{References}

A.G. Hamilton. (1978). Logic for Mathematicians. London: Cambridge University Press, 1978.

D.W.Pei and G.J.Wang, (2002). The completeness of the formal system L_, Science in China (E) (in Chinese), 32(1) (2002): 56-64.

G.J.Wang, and L.Fu. (2005). unified form of triple I method, Computers and Mathematics with Applications, 49(2005): 923-932.

G.J.Wang, L.Fu and J.S.Song. (2002). Theory of truth degrees of propositions in two valued logic, Science in China (A), 45(9) (2002):1106-1116.

G.J.Wang, Yee Leung. (2003). Integrated semantics and logic metric spaces. Fuzzy Sets and Systems, 36 (1)(2003): 71-91.

G.J.Wang. (2003). Introduction of mathematics Logics and resolution principle (in Chinese). Beijing: Science in China Press, 2003.

G.J.Wang. (2003). Non-classical Logics and Approximate Reasoning (in Chinese). Beijing: Science in China Press, 2003

G.J.Wang. (1999). On the logic foundation of fuzzy reasoning. Information Sciences, 117(1)(1999): 47-88.

G.J.Wang. (1998). Theory of tautologies in revised Kleene systems. Science in China, 41(1998): 188-195.

P.H'ajek, (1998). Metamathematics of Fuzzy Logic, Dordrecht: Kluwer Academic Press. 1998.

S.M.Wang, B.S.Wang and G.J.Wang, (2003). A triangular-norm-based propositional fuzzy logic, Fuzzy Sets and Systems, 136(1)(2003):55-70. 